\title{
Woven Structured Triboelectric Nanogenerator for Wearable Devices
}

\author{
Tao Zhou, ${ }^{\dagger}$ Chi Zhang, ${ }^{\dagger}$ Chang Bao Han, ${ }^{\dagger}$ Feng Ru Fan, ${ }^{\dagger}$ Wei Tang, ${ }^{\dagger}$ and Zhong Lin Wang*, ${ }^{\dagger}, \ddagger$ \\ ${ }^{\dagger}$ Beijing Institute of Nanoenergy and Nanosystems, Chinese Academy of Sciences, Beijing, 100083, China \\ ${ }^{\ddagger}$ School of Material Science and Engineering, Georgia Institute of Technology, Atlanta, Georgia 30332, United States
}

\section{Supporting Information}

ABSTRACT: To date, quite a few wearable electronics have entered the market, which are changing the life pattern of consumers. However, the limited lifetime and energy storage capacity have made rechargeable batteries the bottleneck in wearable technology, especially with the increase of number of wearable devices and their large distribution. To solve this problem, we demonstrate a woven-structured triboelectric nanogenerator (W-TENG) using commodity nylon fabric, polyester fabric, and conductive silver fiber fabric. With the advantage of being flexible, washable, breathable, wearable, and able to be triggered by a freestanding triboelectric layer, this W-TENG can move freely without any constraint and is suitable for wearable electronics. To demonstrate the potential applications of the $\mathrm{W}$ TENG, the W-TENG is integrated into shoes, coats, and trousers to harvest different kinds of mechanical energy from human motion. This work presents a new approach in applying triboelectric nanogenerator to wearable devices.

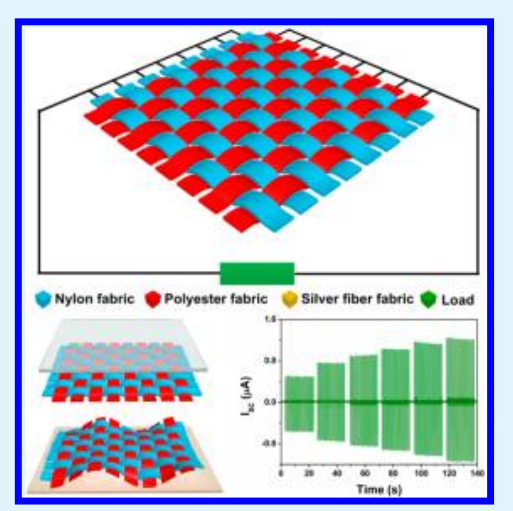

KEYWORDS: triboelectric nanogenerator, energy harvesting, wearable devices, human motion

\section{INTRODUCTION}

In recent years, the concept of "wearable electronics"1,2 has brought a significant paradigm shift in consumer electronics because they can fulfill a variety of functions that are related to our every moment needs, such as taking photos, making phone calls, surfing the Internet, listening to music, monitoring physical health, and so forth. ${ }^{3}$ Wearable electronics is likely to open up a new life pattern for consumers, since they can be integrated with clothes, ${ }^{4,5}$ eye glasses, ${ }^{6}$ wristwatches ${ }^{7}$ and even skin sensors, ${ }^{8,9}$ all of which can be integrated with a cell phone to form a local network using the cell phone as the hub, through which the local network can be linked with Internet. To date, quite a few wearable electronics have entered the market, for example Google Glasses, Apple Smart Watch, Adidas MiCoach, "Hi-call” Bluetooth enabled phone glove, and Cute-circuit Galaxy LED Dress. ${ }^{10}$ One common characteristic of these electronics is that they must be powered by rechargeable batteries, which largely limit their lifetime and sustainable operation. ${ }^{11,12}$ The situation becomes worse if the number of mobile electronics and their distribution are rather huge, making the replacement or charging of batteries almost impossible. Therefore, we anticipate to make these devices, or some of them at least, self-powered by harvesting energy from human body motions, which is ideally suited for wearable electronics.

The most abundant energy from a human motion is mechanical energy. The recently invented triboelectric nanogenerator (TENG) aims at harvesting human motion energy, with the advantages of high efficiency, high reliability, low cost, and environmental friendliness. ${ }^{13-19}$ It operates by a conjunction of triboelectrification and electrostatic induction. ${ }^{15}$ By now, many applications have been demonstrated, such as a kind of power-generating shoe insole ${ }^{20,21}$ to harvest energy from human walking, a TENG built inside clothes ${ }^{22}$ to harvest energy from body motion, and a fiber-based generator ${ }^{5}$ to detect human motion, all of which are based on the relative position change between the two triboelectric materials. Generally, these generators choose metal or carbon as the electrodes and polymer film as the triboelectric materials, making them unwashable, stuffy, and incompatible with clothes.

Herein, we developed a woven-structured triboelectric nanogenerator (W-TENG) based on a freestanding triboelectric-layer mode ${ }^{23}$ to scavenge energy from human motion. In the proposed W-TENG, the positive and negative electrodes are woven together; a freestanding triboelectric layer that moves on the surface of the W-TENG is used to generate electricity. In order to make the W-TENG flexible, wearable, and air permeable enough, commodity nylon fabric and polyester fabric are selected as the two triboelectric-layers, and homemade conductive silver fiber fabric is chosen as the electrodes. At first, the working principle of a textile based freestanding triboelectric-layer TENG (FTENG) is analyzed in order to fully understand the power generating process of the $\mathrm{W}$-TENG. Then, the W-TENG is tested under different conditions (deformation and nondeformation mode) to analyze its electrical output performance. At last, the W-TENG is applied in wearable electronics to harvest different kinds of mechanical energy from human body (walking, movement of joints, shaking, or deformation of clothes, etc.).

Received: June 25, 2014

Accepted: July 27, 2014

Published: July 27, 2014 


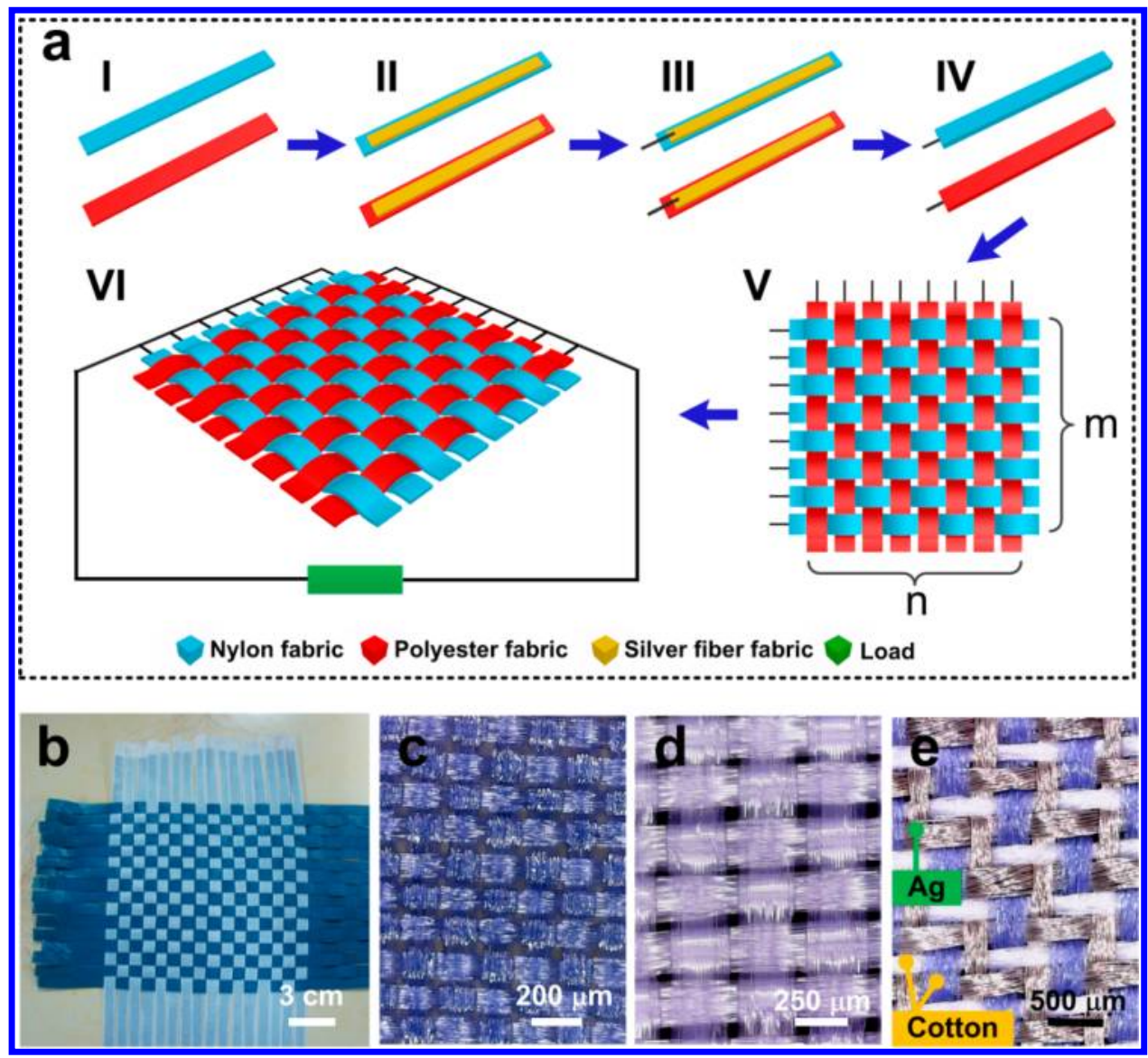

Figure 1. Structure and fabrication of the woven-structured triboelectric nanogenerator (W-TENG). (a) Schematic diagram illustrating the fabricating process of a W-TENG. (b) Digital photography of a $16 \times 16$ W-TENG. (c-e) Electron optical microscope images of the (c) nylon fabric, (d) polyester fabric, and (e) silver fiber fabric.

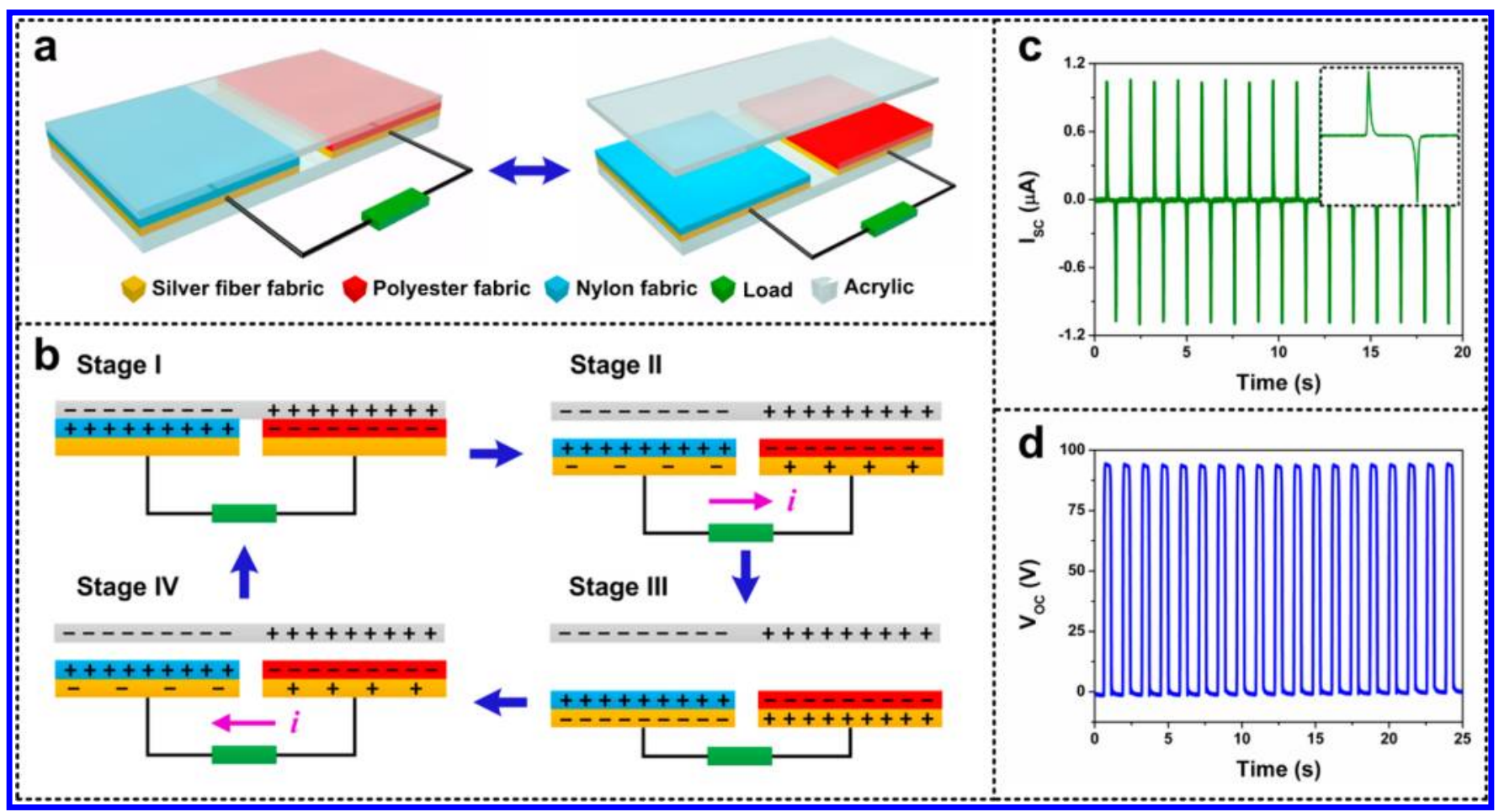

Figure 2. Structure, working mechanism, and electrical outputs of the textile based freestanding triboelectric-layer TENG (FTENG) under vertical contact-separation mode. (a) Structure and working process of the FTENG, where acrylic works as the freestanding triboelectric-layer. (b) Schematic working principle of the FTENG. Electrical outputs of the FTENG: (c) short-circuit current $\left(I_{\mathrm{SC}}\right)$, $(\mathrm{d})$ open-circuit voltage $\left(V_{\mathrm{OC}}\right)$. 


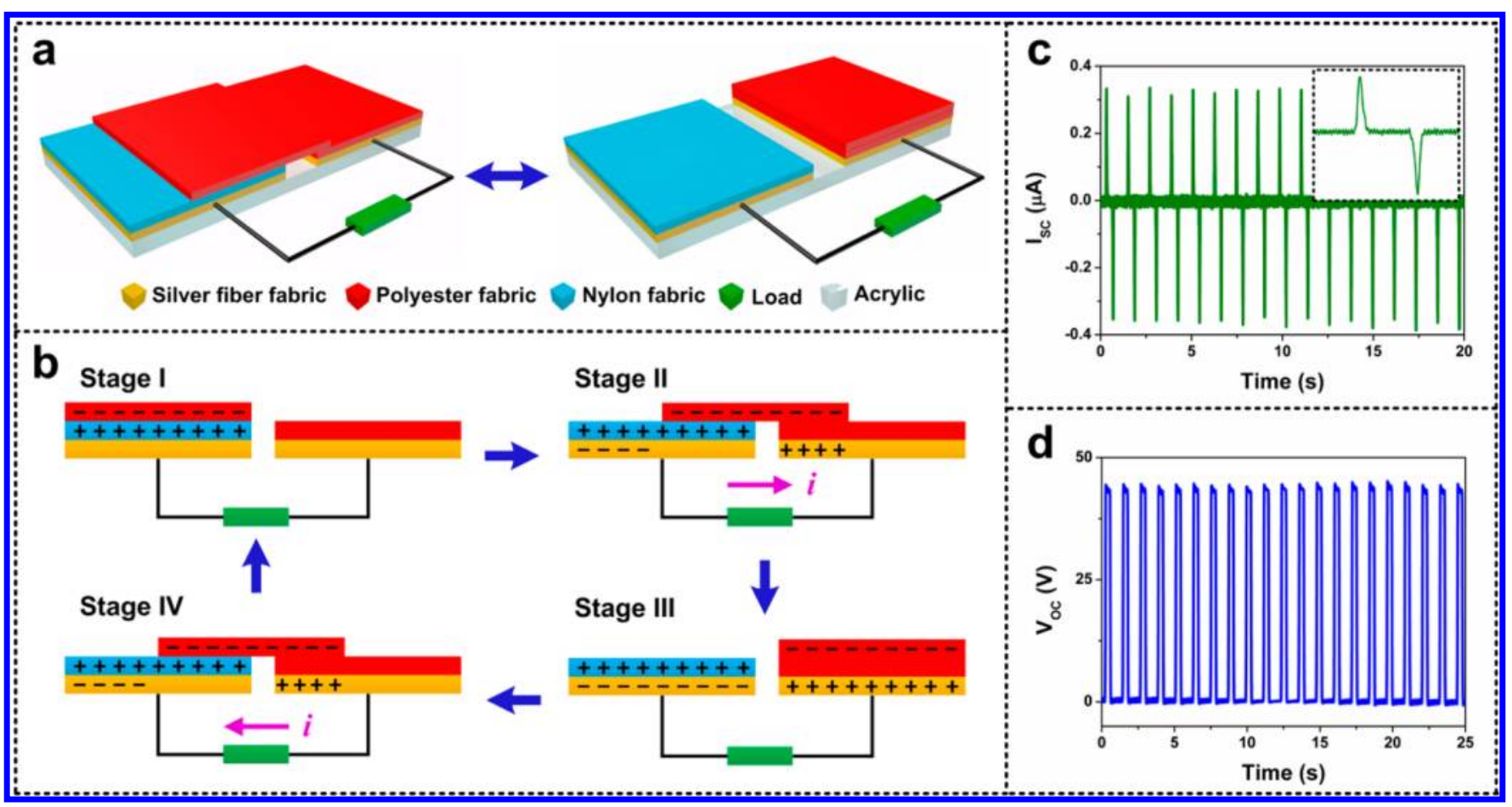

Figure 3. Structure, working mechanism, and electrical outputs of the textile based FTENG under lateral sliding mode. (a) Structure and working process of the FTENG, where polyester fabric works as the freestanding triboelectric-layer. (b) Schematic working principle of the FTENG. Electrical outputs of the FTENG: (c) short-circuit current $\left(I_{\mathrm{SC}}\right),(\mathrm{d})$ open-circuit voltage $\left(V_{\mathrm{OC}}\right)$.

\section{EXPERIMENTAL SECTION}

2.1. Fabrication of the W-TENG. The source materials for the WTENG are commodity nylon fabric $(50 \mu \mathrm{m}$-thick), polyester fabric $(80$ $\mu$ m-thick), and homemade conductive silver fiber fabric (135 m-thick). Electron optical microscopy images of them are shown in Figure 1c-e. Silver fiber fabric that served as the electrodes is a kind of mixed textile made of $\mathrm{Ag}$ fibers and cotton fibers (Figure 1e). Since it is washable, flexible, and highly conductive $\left(0.3 \Omega / \mathrm{cm}^{2}\right)$, silver fiber fabric is more suitable for wearable TENGs than conventional metal electrodes or carbon electrodes. Figure 1a shows the detailed fabrication process of the W-TENG. First, the nylon fabric and polyester fabric are cut into strips ( $7 \mathrm{~mm}$ in width and $29 \mathrm{~cm}$ in length), and silver fiber fabric is cut into $5 \mathrm{~mm} \times 27 \mathrm{~cm}$ strips (Figure 1a I). Second, the pretreated silver fiber fabric strip is pasted onto the center of the nylon fabric strip using acrylic double-sided adhesive (Figure 1a II). Third, a lead wire is connected to the silver fiber fabric by aluminum tape (Figure 1a III). Fourthly, another nylon fabric strip is pasted onto the silver fiber fabric strip, which forms a nylon electrode ( $360 \mu$ m-thick) with silver fiber fabric inside and nylon fabric outside, as shown in Figure 1a IV. Here, the width and length of the silver fiber fabric is less than that of nylon fabric so that it can be fully wrapped by nylon fabric, avoiding the edge leakage of electricity. The fabrication process of the polyester electrode ( $460 \mu \mathrm{m}$-thick) is similar to that of nylon electrode (Figure 1a II-IV). Then, the prepared nylon electrodes and polyester electrodes are woven together (Figure 1a V); suppose the numbers of the nylon electrodes and polyester electrodes are $M$ and $N$, respectively. At last, the nylon electrodes are connected in parallel and the polyester electrodes in parallel, too; then; a $M \times N$ W-TENG is completed (Figure $1 \mathrm{a} \mathrm{VI})$. Figure $1 \mathrm{~b}$ shows a digital photography of a $16 \times 16$ W-TENG.

2.2. Measurement of the TENGs. The short-circuit current of the TENGs was measured by a low noise current preamplifier (Stanford Research Systems, SR570). The open-circuit voltage and transferred charge were measured by an electrometer with very large input resistance (Keithley, 6517B). A mechanical linear motor (Linmot, E1100) was employed to drive the TENGs at different velocities, accelerations, and displacements, as illustrated in Supporting Information (SI) Figure S1.

\section{RESULT AND DISCUSSION}

In order to fully understand the working mechanism of the proposed W-TENG, a textile based FTENG is analyzed at first. In the FTENG, nylon fabric $(5 \mathrm{~cm} \times 5 \mathrm{~cm})$ and polyester fabric $(5 \mathrm{~cm} \times 5 \mathrm{~cm})$ act as the two triboelectric surfaces, and two pieces of silver fiber fabric $(5 \mathrm{~cm} \times 5 \mathrm{~cm})$ act as the electrodes. These materials are adhered onto a piece of acrylic plate $(5 \mathrm{~cm}$ $\times 11 \mathrm{~cm}$ ), which works as the supporting substrate (Figures $2 \mathrm{a}$ and $3 \mathrm{a})$. According to previous research, there are two working modes for the FTENG: vertical contact-separation mode ${ }^{15-19}$ and lateral sliding mode. ${ }^{18,19,23}$

The structure of the FTENG under vertical contactseparation mode is shown in Figure $2 \mathrm{a}$, where a piece of acrylic plate $(5 \mathrm{~cm} \times 11 \mathrm{~cm})$ is chosen as the freestanding triboelectric-layer. Driven by a vertical force, the freestanding acrylic plate will contact and separate from the FTENG periodically and thus generate alternating-current in the load. At first, the freestanding acrylic plate is brought into full contact with the FTENG (Figure 2b Stage I). Supposing the freestanding acrylic plate and the FTENG are uncharged beforehand, the triboelectric effect will render nylon surface with positive charges, the part of the acrylic that contacts with the nylon fabric with negative charges, polyester surface with negative charges, and the part of the acrylic that contacts with the polyester fabric with positive charges, as shown in Figure $2 b$ Stage I. When the freestanding acrylic plate starts to move apart from the FTENG, an electric potential difference is produced, which drives electrons to flow from the polyester electrode to the nylon electrode through an external circuit in order to balance the generated triboelectric potential (Figure 2b Stage II). When the separation between the freestanding acrylic plate and the FTENG is maximized, the flow of electrons stopped because an electrostatic equilibrium is reached (Figure $2 \mathrm{~b}$ Stage III). Subsequently, the freestanding acrylic plate is driving to 


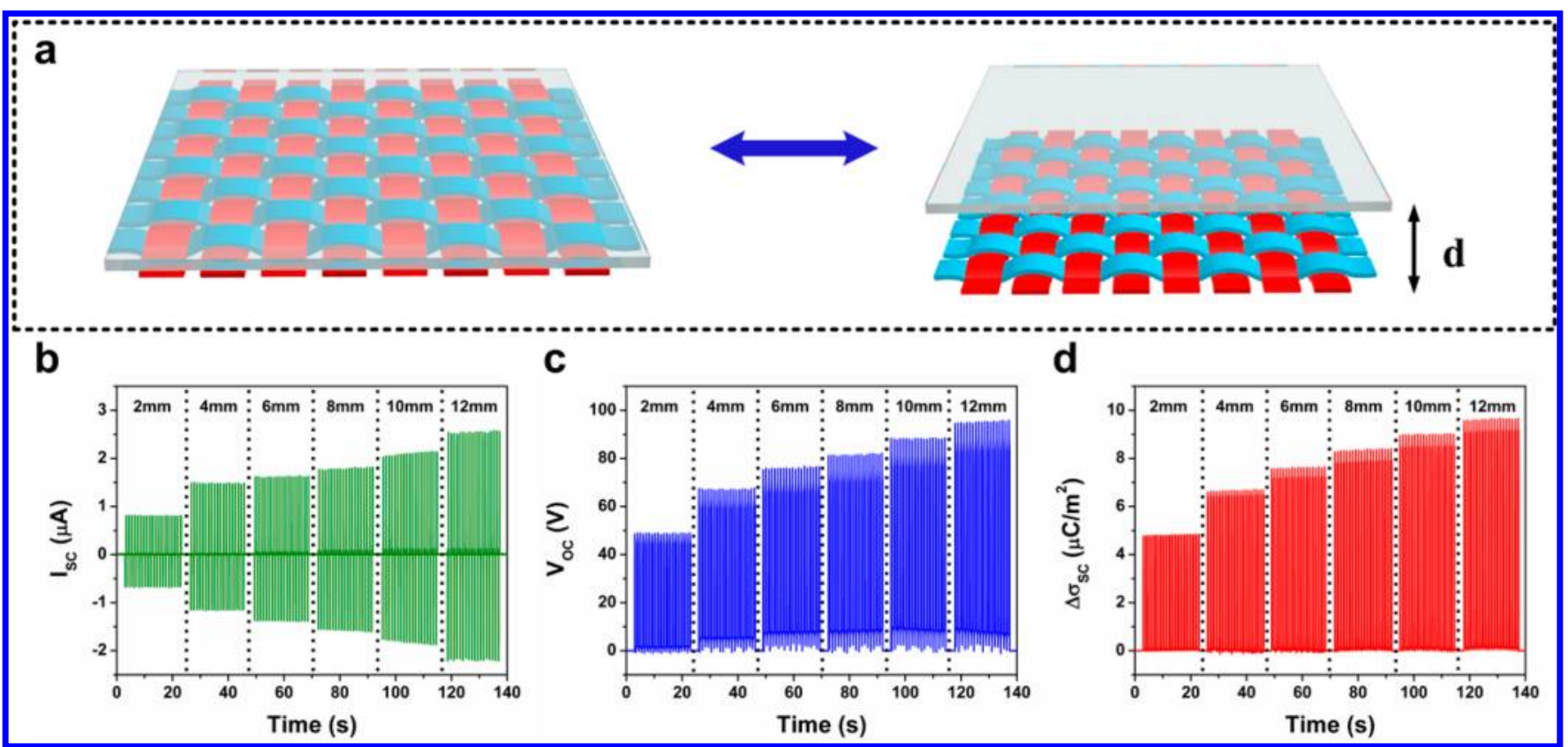

Figure 4. Structure and electrical outputs of the W-TENG under nondeformation mode. (a) Structure and working process of the W-TENG, in which acrylic is chosen as the freestanding triboelectric layer. $(\mathrm{b}-\mathrm{d})$ The measured short-circuit current $\left(I_{\mathrm{SC}}\right)$, open-circuit voltage $\left(V_{\mathrm{OC}}\right)$ and transferred charge density $\left(\Delta \sigma_{\mathrm{SC}}\right)$ of a $16 \times 16 \mathrm{~W}$-TENG under different distance $(d=2,4,6,8,10,12 \mathrm{~mm})$.

contact with the FTENG again, breaking the former electrostatic equilibrium. In consequence, electrons flow from nylon electrode back to polyester electrode, reducing the amount of induced charges (Figure $2 \mathrm{~b}$ Stage IV). When the freestanding acrylic plate and the FTENG contact with each other again, all of the induced charges are neutralized (Figure $2 b$ Stage I). To measure the electrical outputs of the FTENG, the displacement, acceleration, and maximum speed of the linear motor were set as $40 \mathrm{~mm}, 10 \mathrm{~m} / \mathrm{s}^{2}$ and $20 \mathrm{~m} / \mathrm{s}$, respectively. In this situation, the FTENG could generate a short-circuit current $\left(I_{\mathrm{SC}}\right)$ up to $1 \mu \mathrm{A}$ and an open-circuit voltage $\left(V_{\mathrm{OC}}\right)$ about $90 \mathrm{~V}$ (Figure $2 c$ and $d$ ). The inset in Figure $2 c$ is an enlarged view of the $I_{\mathrm{SC}}$ in one cycle that validates the working principle described in Figure $2 b$.

Similarly, the structure of the FTENG under lateral sliding mode is shown in Figure 3a, where polyester fabric $(5 \mathrm{~cm} \times 5$ $\mathrm{cm})$ acts as the freestanding triboelectric-layer. ${ }^{23,24}$ At first, the freestanding polyester fabric is brought into contact with the nylon electrode (Figure 3b Stage I). Because polyester is much more triboelectrically negative than nylon, electrons will transfer from nylon into the freestanding polyester fabric. When the freestanding polyester fabric slides toward the polyester electrode (Figure 3b Stage II), electrons will flow from polyester electrode to nylon electrode. The electrons stop flow when the freestanding polyester fabric completely overlaps with the polyester electrode (Figure 3b Stage III). Here, we suppose that the static charges on the freestanding polyester fabric will not transfer to the surface of the polyester electrode in order to simplify the working principle. Subsequently, a backward sliding of the freestanding polyester fabric from the polyester electrode to the nylon electrode drives the flow of electrons in the opposite direction (Figure 3b Stage IV). When the freestanding polyester fabric overlaps with the nylon electrode again, the flow of electrons stopped (Figure 3b Stage I). As shown in Figure $3 \mathrm{c}$ and $\mathrm{d}$, the maximum $I_{\mathrm{SC}}$ and $V_{\mathrm{OC}}$ of the FTENG is about $0.35 \mu \mathrm{A}$ and $45 \mathrm{~V}$ where the acceleration and maximum speed of the linear motor were $30 \mathrm{~m} / \mathrm{s}^{2}$ and 30 $\mathrm{m} / \mathrm{s}$.

A woven structured TENG (W-TENG) is proposed in order to apply the above textile based FTENG in wearable devices and harvest energy from human motion. The structure and fabrication process of the W-TENG have been described in Experimental Section. The proposed W-TENG could be easily integrated to clothes, shoes, gloves, and carpets, because it is flexible, wearable, washable, and air permeable enough. In general, the W-TENG has two basic working modes: deformation and nondeformation mode. In the nondeformation mode (Figure 4a), the W-TENG is adhered onto a piece of acrylic plate, which works as the supporting substrate. Another piece of acrylic plate that acts as the freestanding triboelectric layer is driven by a vertical force to contact with and separate from the W-TENG periodically. The working mechanism of the W-TENG under nondeformation mode is similar to Figure $2 \mathrm{~b}$. To analyze the output capability of the W-TENG, a linear motor with a acceleration of $50 \mathrm{~m} / \mathrm{s}^{2}$ and a maximum speed of $1.2 \mathrm{~m} / \mathrm{s}$ is employed to trigger the $16 \times 16 \mathrm{~W}$-TENG, wherein the maximum distance $(d)$ between the freestanding acrylic plate and the W-TENG is purposely set at 2, 4, 6, 8, 10, and 12 $\mathrm{mm}$. As shown in Figure $4 \mathrm{~b}-\mathrm{d}$, when $d$ varies from 2 to $12 \mathrm{~mm}$, the maximum short-circuit current $\left(I_{\mathrm{SC}}\right)$, open-circuit voltage $\left(V_{\mathrm{OC}}\right)$,and transferred charge density $\left(\Delta \sigma_{\mathrm{SC}}\right)$ increased from $0.8 \mu \mathrm{A}, 48 \mathrm{~V}, 4.7 \mu \mathrm{C} / \mathrm{m}^{2}$ to $2.5 \mu \mathrm{A}, 95 \mathrm{~V}, 9.6 \mu \mathrm{C} / \mathrm{m}^{2}$. In other words, the increase of $d$ is favorable for the magnitude of $I_{\mathrm{SC}}$, $V_{\mathrm{OC}}$ and $\Delta \sigma_{\mathrm{SC}}$. The influence of acceleration on the outputs of $\mathrm{W}$-TENG under nondeformation mode is shown in SI Figure $\mathrm{S} 2 \mathrm{a}-\mathrm{c}$.

In the deformation mode of the W-TENG (Figure 5a), a pieces of polyester fabric is selected as the freestanding triboelectric layer and is pasted onto the supporting substrate made of acrylic. Then, the left margin of the W-TENG is fixed to the aforesaid freestanding polyester substrate; meanwhile, the right margin of the W-TENG is fixed to the linear motor that could provide a lateral force to stretch and compress the 


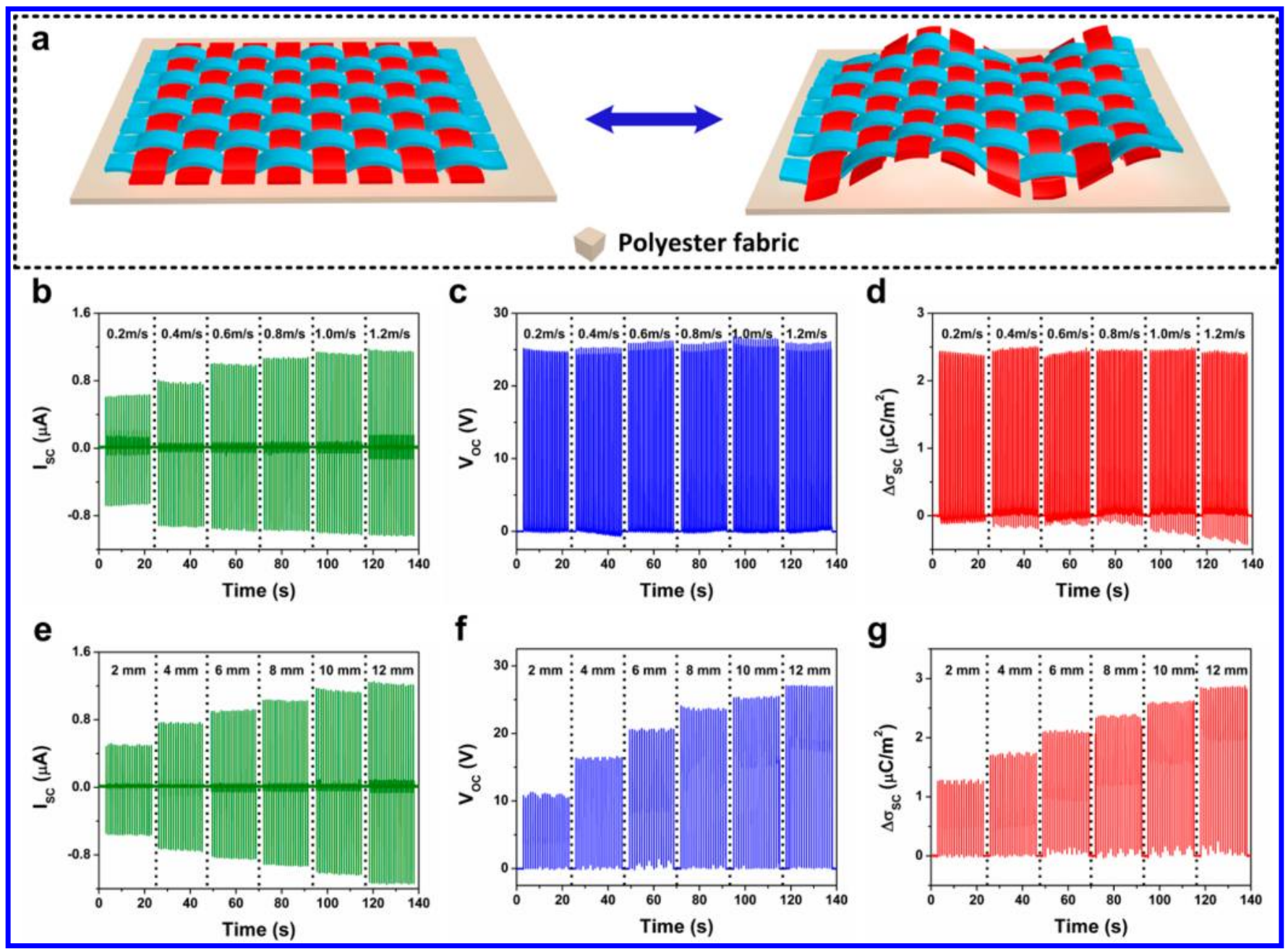

Figure 5. Structure and electrical outputs of the W-TENG under deformation mode. (a) Structure and working process of the W-TENG, where polyester fabric is chosen as the freestanding triboelectric layer. $(\mathrm{b}-\mathrm{d})$ The short-circuit current $\left(I_{\mathrm{SC}}\right)$, open-circuit voltage $\left(V_{\mathrm{OC}}\right)$, and transferred charge density $\left(\Delta \sigma_{\mathrm{SC}}\right)$ of a $16 \times 16 \mathrm{~W}$-TENG under different deformation speed $(0.2,0.4,0.6,0.8,1.0,1.2 \mathrm{~m} / \mathrm{s})$. (e-g) The short-circuit current $\left(I_{\mathrm{SC}}\right)$, open-circuit voltage $\left(V_{\mathrm{OC}}\right)$, and transferred charge density $\left(\Delta \sigma_{\mathrm{SC}}\right)$ of a $16 \times 16 \mathrm{~W}$-TENG under different displacement $(2,4,6,8,10,12 \mathrm{~mm})$.

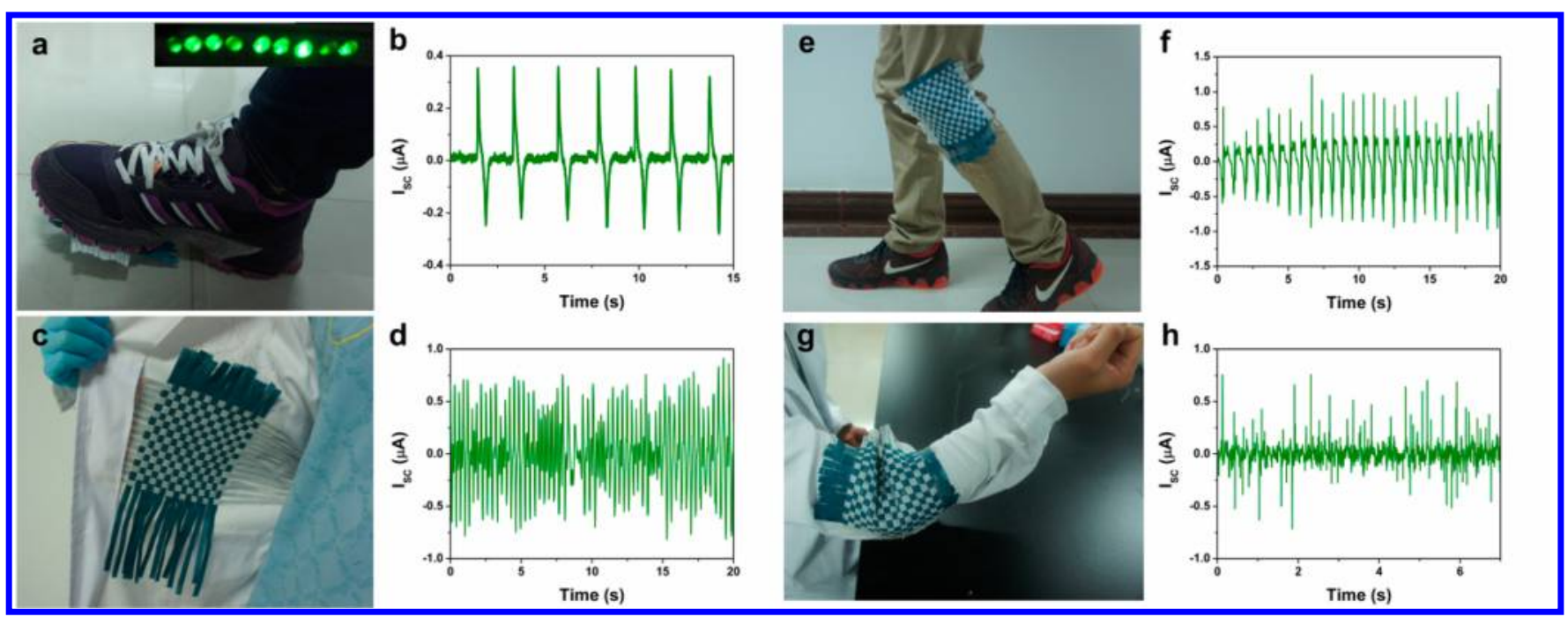

Figure 6. Applications of the W-TENG to harvest different kinds of mechanical energy from human body. (a) A power generating shoe (P-shoe) that could harvest energy from footsteps and light up about 9 LEDs. (b) The current generated by the P-shoe. (c) A W-TENG integrated in a coat harvesting energy from the shaking of clothes. (d) The current generated by the coat. (e) The W-TENG harvesting energy from leg joints bend. (f) The current generated by the leg joint. (g) The W-TENG harvesting energy from arm joints. (h) The current generated by the arm joint. 
W-TENG periodically, forming a lateral deformation of the WTENG. During the process of the deformation, the factual contact area between the W-TENG and the freestanding polyester substrate will decrease and increase alternatively, generating an alternating current in the load. The working principle of the W-TENG under deformation mode can refer to Figures $2 \mathrm{~b}$ and $3 \mathrm{~b}$ for understanding where the freestanding triboelectric layer is polyester rather than acrylic. In order to adequately study the factors that affect the outputs of the WTENG, the $16 \times 16 \mathrm{~W}$-TENG is tested under different conditions. First, the W-TENG is measured under different deformation speed. Namely, the acceleration and displacement of the linear motor are set as $50 \mathrm{~m} / \mathrm{s}^{2}$ and $10 \mathrm{~mm}$; the speed of the linear motor is set as $0.2,0.4,0.6,0.8,1.0$, and $1.2 \mathrm{~m} / \mathrm{s}$. As shown in Figure $5 \mathrm{~b}-\mathrm{d}$, when the speed increases from 0.2 to $1.2 \mathrm{~m} / \mathrm{s}, V_{\mathrm{OC}}$ and $\Delta \sigma_{\mathrm{SC}}$ almost remain the same $(25 \mathrm{~V}$ and 2.4 $\left.\mu \mathrm{C} / \mathrm{m}^{2}\right)$, while the amplitude of $I_{\mathrm{SC}}$ increases dramatically from $0.6 \mu \mathrm{A}$ to $1.2 \mu \mathrm{A}$, which means that the increase of speed has no influence on $V_{\mathrm{OC}}$ and $\Delta \sigma_{\mathrm{SC}}$, but it leads to an increase of $I_{\mathrm{SC}}$. Second, the W-TENG is measured under different deformation displacement. The acceleration and speed of the linear motor are set as $50 \mathrm{~m} / \mathrm{s}^{2}$ and $1.2 \mathrm{~m} / \mathrm{s}$, and the displacement of the linear motor is set as $2,4,6,8,10$, and $12 \mathrm{~mm}$.

As shown in Figure $5 \mathrm{e}-\mathrm{g}$, as the displacement increases from 2 to $12 \mathrm{~mm}, I_{\mathrm{SC}}, V_{\mathrm{OC}}$, and $\Delta \sigma_{\mathrm{SC}}$ increase from $0.5 \mu \mathrm{A}, 11 \mathrm{~V}$, and $1.2 \mu \mathrm{C} / \mathrm{m}^{2}$ to $1.2 \mu \mathrm{A}, 27 \mathrm{~V}$, and $2.8 \mu \mathrm{C} / \mathrm{m}^{2}$. That is to say, the increase of deformation displacement is favorable for the magnitude of $I_{\mathrm{SC}}, V_{\mathrm{OC}}$, and $\Delta \sigma_{\mathrm{SC}}$. The influence of acceleration on the outputs of W-TENG under deformation mode is shown in SI Figure S2d-f.

Being flexible, washable, breathable, wearable, and able to be triggered by a freestanding triboelectric layer, this W-TENG can move freely without any constraint, which will largely expand the applications of TENG for versatile mechanical energy harvesting, especially for wearable devices. Besides, other than acrylic and polyester, the freestanding triboelectric layer could also be cotton, wool, paper, polymer, the ground, and so on. To demonstrate the potential applications of the $\mathrm{W}$ TENG, it is integrated to shoes, coats, and trousers to harvest different kinds of mechanical energy from human body (Figure 6). To harvest energy from footsteps, an W-TENG with an effective area of $20 \mathrm{~cm}^{2}$ is pasted onto the bottom of a sports shoe, making up a power generating shoe (P-shoe) that is capable of instantaneously lighting up about 9 LEDs (Figure $6 a)$. Here, a piece of acrylic plate is laid on the ground acting as the freestanding triboelectric layer, which corresponds to the nondeformation mode. Figure $6 \mathrm{~b}$ shows the current generated by the P-shoe, which reaches more than $0.3 \mu \mathrm{A}$. Since each footstep can generate one cycle of current, the W-TENG could also work as active sensor that calculates the number of footsteps. The W-TENG could also be integrated in coats to harvest energy from the shaking of clothes (Figure 6c). Figure $6 \mathrm{~d}$ shows the current generated by the power generating coat, which reaches about $0.75 \mu \mathrm{A}$. As we know, the movement of legs, arms, and hands is one of the most important mechanical energy supplied by human body. If we integrate the W-TENG in trousers, we can harvest energy from the bending of leg joints (Figure 6e). Here, the trousers made of polyester fabric acts as the freestanding triboelectric layer. When one walks around, the movement of leg joints will lead to a changing of the contact area between the W-TENG and the trousers generating an alternating current of about $0.9 \mu \mathrm{A}$ (Figure 6f), corresponding to the deformation mode. The W-TENG could also be integrated into coats in order to harvest energy from the movement of arm joints (Figure $6 \mathrm{~g}$ ). Figure $6 \mathrm{~h}$ shows that the bending of arm can generate an alternating current of about $0.75 \mu \mathrm{A}$. As mentioned above, the proposed W-TENG is an extremely promising power supply for wearable devices. Such structure can also be extended to other applications.

\section{CONCLUSION}

In summary, this paper demonstrates a new woven structured triboelectric nanogenerator based on commonly used textiles (nylon and polyester fabrics). In order to make the TENG washable and flexible enough, conductive silver fiber fabric is selected as the electrodes. Different from other TENG, electricity is generated by a freestanding triboelectric-layer that moves relatively on the surface of the W-TENG rather than the relative movement between the positive and negative electrodes. The working principle of a textile-based freestanding triboelectric-layer TENG (FTENG) is analyzed at first in order to fully understand the power generating process of the $\mathrm{W}$ TENG. The FTENG could work under both vertical contactseparation mode and lateral sliding mode, while the W-TENG can work under nondeformation mode and deformation mode that is quite suitable for wearable electronics. From both theoretical analysis and experimental demonstration, the $\mathrm{W}$ TENG is proven to be very effective in harvesting different kinds of mechanical energy from human body, such as footsteps, shaking of clothes, and the bending of legs and arms. Being flexible, washable, breathable, and wearable, the newly designed W-TENG provides a new solution for the power supply of wearable devices.

\section{ASSOCIATED CONTENT}

\section{S Supporting Information}

Schematic diagram illustrating the TENGs (FTENG and WTENG) driven by a linear motor under different working modes (vertical contact-separation, lateral sliding, deformation, and nondeformation modes); influences of the acceleration on the outputs of the W-TENG in both the deformation and nondeformation mode. This material is available free of charge via the Internet at http://pubs.acs.org.

\section{AUTHOR INFORMATION}

\section{Corresponding Author}

*Email: zlwang@gatech.edu.

\section{Notes}

The authors declare no competing financial interest.

\section{ACKNOWLEDGMENTS}

This research was supported by the "thousands talents" program for pioneer researcher and his innovation team, China, and Beijing City Committee of science and technology projects (Z131100006013004, Z131100006013005).

\section{REFERENCES}

(1) Kou, L.; Huang, T.; Zheng, B.; Han, Y.; Zhao, X.; Gopalsamy, K.; Sun, H.; Gao, C. Coaxial Wet-Spun Yarn Supercapacitors for HighEnergy Density and Safe Wearable Electronics. Nat. Commun. 2014, 5, $1-10$.

(2) Son, D.; Lee, J.; Qiao, S.; Ghaffari, R.; Kim, J.; Lee, J. E.; Song, C.; Kim, S. J.; Lee, D. J.; Jun, S. W.; Yang, S.; Park, M.; Shin, J.; Do, K.; Lee, M.; Kang, K.; Hwang, C. S.; Lu, N.; Hyeon, T.; Kim, D. H. Multifunctional Wearable Devices for Diagnosis and Therapy of Movement Disorders. Nat. Nanotechnol. 2014, 9, 397-404. 
(3) Lee, Y. H.; Kim, J. S.; Noh, J.; Lee, I.; Kim, H. J.; Choi, S.; Seo, J.; Jeon, S.; Kim, T. S.; Lee, J. Y.; Choi, J. W. Wearable Textile Battery Rechargeable by Solar Energy. Nano Lett. 2013, 13, 5753-5761.

(4) Meng, Y.; Zhao, Y.; Hu, C.; Cheng, H.; Hu, Y.; Zhang, Z.; Shi, G.; $\mathrm{Qu}$, L. All-Graphene Core-Sheath Microfibers for All-Solid-State, Stretchable Fibriform Supercapacitors and Wearable Electronic Textiles. Adv. Mater. 2013, 25, 2326-2331.

(5) Zhong, J.; Zhang, Y.; Zhong, Q.; Hu, Q.; Hu, B.; Wang, Z. L.; Zhou, J. Fiber-Based Generator for Wearable Electronics and Mobile Medication. ACS Nano 2014, 8, 6273-6280.

(6) Feng, S.; Caire, R.; Cortazar, B.; Turan, M.; Wong, A.; Ozcan, A. Immunochromatographic Diagnostic Test Analysis Using Google Glass. ACS Nano 2014, 8, 3069-9079.

(7) Wile, D. J.; Ranawaya, R.; Kiss, Z. H. Smart Watch Accelerometry for Analysis and Diagnosis of Tremor. J. Neurosci. Methods 2014, 230, $1-4$.

(8) Kim, D. H.; Lu, N.; Ma, R.; Kim, Y. S.; Kim, R. H.; Wang, S.; Wu, J.; Won, S. M.; Tao, H.; Islam, A.; Yu, K. J.; Kim, T. I.; Chowdhury, R.; Ying, M.; Xu, L.; Li, M.; Chung, H. J.; Keum, H.; McCormick, M.; Liu, P.; Zhang, Y. W.; Omenetto, F. G.; Huang, Y.; Coleman, T.; Rogers, J. A. Epidermal Electronics. Science 2011, 333, 838-843.

(9) Wang, X.; Gu, Y.; Xiong, Z.; Cui, Z.; Zhang, T. Silk-Molded Flexible, Ultrasensitive, and Highly Stable Electronic Skin for Monitoring Human Physiological Signals. Adv. Mater. 2014, 26, 1336-1342.

(10) Jost, K.; Dion, G.; Gogotsi, Y. Textile Energy Storage in Perspective. J. Mater. Chem. A 2014, 2, 10776-10787, DOI: 10.1039/ c4ta00203b.

(11) Choi, N. S.; Chen, Z.; Freunberger, S. A.; Ji, X.; Sun, Y. K.; Amine, K.; Yushin, G.; Nazar, L. F.; Cho, J.; Bruce, P. G. Challenges Facing Lithium Batteries and Electrical Double-Layer Capacitors. Angew. Chem., Int. Ed. 2012, 51, 9994-10024.

(12) Tarascon, J. M.; Armand, M. Issues and Challenges Facing Rechargeable Lithium Batteries. Nature 2001, 414, 359-367.

(13) Wang, Z. L. Triboelectric Nanogenerators As New Energy Technology for Self-Powered Systems and As Active Mechanical and Chemical Sensors. ACS Nano 2013, 7, 9533-9557.

(14) Fan, F. R.; Tian, Z. Q.; Wang, Z. L. Flexible Triboelectric Generator. Nano Energy 2012, 1, 328-334.

(15) Zhu, G.; Pan, C.; Guo, W.; Chen, C. Y.; Zhou, Y.; Yu, R.; Wang, Z. L. Triboelectric-Generator-Driven Pulse Electrodeposition for Micropatterning. Nano Lett. 2012, 12, 4960-4965.

(16) Zhong, J.; Zhong, Q.; Fan, F.; Zhang, Y.; Wang, S.; Hu, B.; Wang, Z. L.; Zhou, J. Finger Typing Driven Triboelectric Nanogenerator and its Use for Instantaneously Lighting Up LEDs. Nano Energy 2013, 2, 491-497.

(17) Zhu, G.; Lin, Z. H.; Jing, Q.; Bai, P.; Pan, C.; Yang, Y.; Zhou, Y.; Wang, Z. L. Toward Large-Scale Energy Harvesting by a NanoparticleEnhanced Triboelectric Nanogenerator. Nano Lett. 2013, 13, 847853.

(18) Wang, S.; Lin, L.; Xie, Y.; Jing, Q.; Niu, S.; Wang, Z. L. SlidingTriboelectric Nanogenerators based on In-Plane Charge-Separation Mechanism. Nano Lett. 2013, 13, 2226-2233.

(19) Zhu, G.; Chen, J.; Liu, Y.; Bai, P.; Zhou, Y. S.; Jing, Q.; Pan, C.; Wang, Z. L. Linear-Grating Triboelectric Generator based on Sliding Electrification. Nano Lett. 2013, 13, 2282-2289.

(20) Hou, T. C.; Yang, Y.; Zhang, H.; Chen, J.; Chen, L. J.; Wang, Z. L. Triboelectric Nanogenerator Built Inside Shoe Insole for Harvesting Walking Energy. Nano Energy 2013, 2, 856-862.

(21) Zhu, G.; Bai, P.; Chen, J.; Wang, Z. L. Power-Generating Shoe Insole based on Triboelectric Nanogenerators for Self-Powered Consumer Electronics. Nano Energy 2013, 2, 688-692.

(22) Zhang, H.; Yang, Y.; Hou, T. C.; Su, Y.; Hu, C.; Wang, Z. L. Triboelectric Nanogenerator Built Inside Clothes for Self-Powered Glucose Biosensors. Nano Energy 2013, 2, 1019-1024.

(23) Wang, S.; Xie, Y.; Niu, S.; Lin, L.; Wang, Z. L. Freestanding Triboelectric-Layer-Based Nanogenerators for Harvesting Energy from a Moving Object or Human Motion in Contact and Noncontact Modes. Adv. Mater. 2014, 26, 2818-2824.
(24) Lin, L.; Wang, S.; Niu, S.; Liu, C.; Xie, Y.; Wang, Z. L. Noncontact Free-Rotating Disk Triboelectric Nanogenerator as a Sustainable Energy Harvester and Self-Powered Mechanical Sensor. ACS Appl. Mater. Interfaces 2014, 6, 3031-3038. 